\title{
Automatic Modeling of Anatomical Structures for Biomechanical Analysis and Visualization in a Virtual Spine Workstation
}

\author{
Xuesong Chen ${ }^{1}$, Chee-Kong Chui ${ }^{1 *}$, Swee-Hin Teoh $^{2}$, Sim-Heng Ong ${ }^{2}$, \\ Wieslaw L. Nowinski ${ }^{1}$ \\ 1 Biomedical Lab, Kent Ridge Digital Lab, Singapore \\ * cheekong@krdl.org.sg \\ 2 Laboratory for Biomedical Engineering \& BIOMAT, National University of Singapore, \\ Singapore
}

\section{Introduction}

With the advances in medical imaging, we can now extract quantitative information about human anatomical structures in three dimensions from images of various modalities. But visualization of anatomical structures and finite element analysis are two technical problems for representation of biomedical objects and simulation of physiological behavior. We describe the component of our workstation that contributes to the automatic generation of $3 \mathrm{D}$ physical-based meshes used in biomechanical engineering analysis and visualization of complex anatomical structures. This component extends the provision of visual information of the human spine to physical-based analysis for predicting the effects of treatment. These effects range from instrumentation in deformity correction, treatments on the courses of nerves and the resulting strains to the eventual interaction between bone and implants. One of the first applications of our approach was the used of the physical-based model to investigate the injury to human spine among airforce pilots in high G flying.

\section{Automatic Modeling in the Virtual Spine Workstation}

Figure 1 describes the processing pipeline of the virtual spine workstation. From medical images, after a preprocessing of identification and segmentation, we can start the automatic modeling of anatomical structures, which can generate physical-based meshes for biomechanical analysis and visualization. The automatic model generation process consists of several steps: Multiple Resolution 2D Meshing, 3D Meshing, Optimization and Validation.

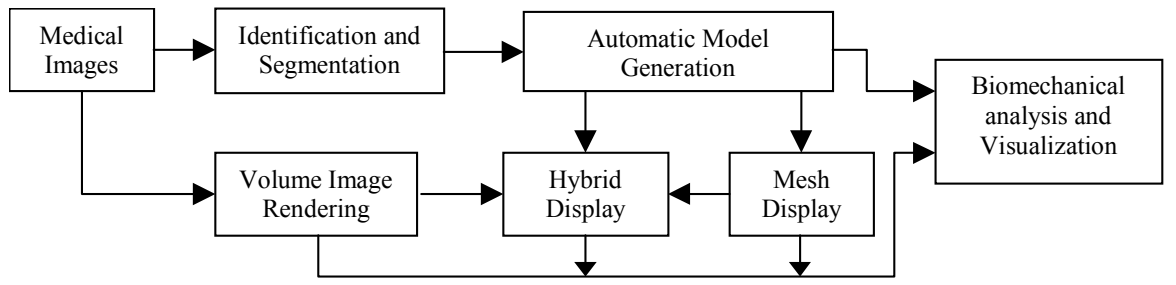

Figure 1. Processing pipeline of the virtual workstation

W. Niessen and M. Viergever (Eds.): MICCAI 2001, LNCS 2208, pp. 1170-1171, 2001.

(C) Springer-Verlag Berlin Heidelberg 2001 
A grid plane approach for 2D meshing at each slice is proposed, which divides the area equally and generates regular elements. Firstly, the region on every slice is gridded at two orthogonal directions with a flexible resolution, which can be changed by the user to perform a better FEM analysis. Then, 2D meshes are generated using a two-dimensional marching cube algorithm. Regular elements of quadrilateral shape are constructed as interior core elements while triangular elements along the boundaries. Secondly, the nodes and elements are numbered to construct an FEM mesh system for engineering analysis.

The 3D meshing process joins 2D planar meshes at two adjacent slices to form volumetric meshes. A 3D grid frame structure establishes the topological relation of any two adjacent grid planes. The grid frame approach depicts the geometrical closeness of the contours at the adjacent slices, provides an accurate and convenient means to identify the topological connection of the anatomical contours. The 3D meshes are therefore built upon the grid frame by connecting corresponding nodes at adjacent grid planes.

Due to the complexity of the object's geometry, optimization and validation processes are performed to standardize the elements at the boundary to comply with the requirement for FEM analysis. In order to identify the related region accurately, the optimization process modifies the local coordinate system and performs adaptive meshing of the local region. After the process, the validation process checks each element by computing Jacobians, aspect ratios, volumes and areas of elements to meet the requirements of the FEM analysis software.

\section{Results and Discussions}

The generated 3D meshes of the anatomical structures allow for biomechanical analysis and visualization. Figure 2(a) shows the result of axial compression of L4 vertebrae after FEM analysis using ABAQUS packages. Figure 2(b) is a visualization of a human spine with the generated 3D meshes from VHD data [1]. Our modeling technique is applied to other anatomical structures. We are extending this workstation for clinicians to perform pre-operative planning for image guided spinal surgery.

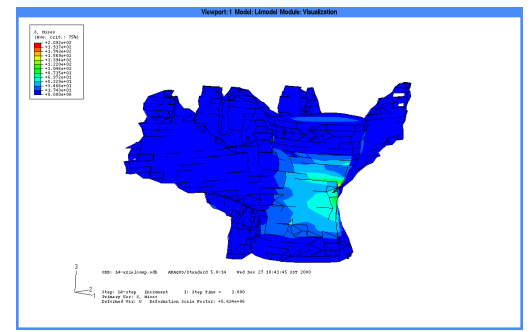

(a)

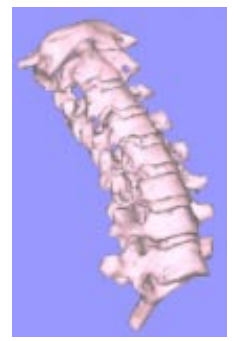

(b)

Figure 2. Biomechanical analysis and visualization with the 3D meshes

\section{References}

1. Spitzer VM, Whitelock DG (1998). National Library of Medicine, Atlas of the Visible Human Male: Reverse Engineering of the Human Body. Jones and Bartlett Publishers. 